\title{
A novel cause of mild/moderate hemophilia A: mutations scattered in the factor VIII C1 domain reduce factor VIII binding to von Willebrand factor
}

\author{
Marc Jacquemin, Renaud Lavend'homme, Abdellah Benhida, Beatrijs Vanzieleghem, Roseline d'Oiron, Jean-Maurice Lavergne, \\ Hans H. Brackmann, Rainer Schwaab, Thierry VandenDriessche, Marinee K. L. Chuah, Marc Hoylaerts, Jean Guy G. Gilles, \\ Kathelijne Peerlinck, Jos Vermylen, and Jean-Marie R. Saint-Remy
}

\begin{abstract}
The mechanisms responsible for the low factor VIII (fVIII) activity in the plasma of patients with mild/moderate hemophilia A are poorly understood. In such patients, we have identified a series of fVIII mutations (lle2098Ser, Ser2119Tyr, Asn2129Ser, Arg2150His, and Pro2153GIn) clustered in the $\mathbf{C} 1$ domain and associated with reduced binding of $\mathrm{fVIII}$ to von Willebrand factor (vWf). For each patient plasma, the specific activity of mutated fVIII was close to that of normal fVIII. Scatchard analysis showed that the affinity for vWf of recombinant Ile2098Ser, Ser2119Tyr, and Arg2150His fVIII mutants was re-
\end{abstract}

duced 8-fold, 80-fold, and 3-fold, respectively, when compared with normal fVIII. Given the importance of vWf for the stability of fVIII in plasma, these findings suggested that the reduction of $\mathrm{fVIII}$ binding to vWf resulting from the abovementioned mutations could contribute to patients' low fVIII plasma levels. We, therefore, analyzed the effect of vWf on fVIII production by Chinese hamster ovary (CHO) cells transfected with expression vectors for recombinant $B$ domaindeleted normal, Ile2098Ser, Ser2119Tyr, and Arg2150His fVIII. These 3 mutations impaired the vWf-dependent accumula- tion of functional fVIII in culture medium. Analysis of fVIII production by transiently transfected $\mathrm{CHO}$ cells indicated that, in addition to the impaired stabilization by vWf, the secretion of functional lle2098Ser and Arg2150His fVIII was reduced about 2-fold and 6-fold, respectively, by comparison to Ser2119Tyr and normal fVIII. These findings indicate that $\mathrm{C} 1$-domain mutations resulting in reduced fVIII binding to vWf are an important cause of mild/ moderate hemophilia A. (Blood. 2000;96: 958-965)

( 2000 by The American Society of Hematology

\section{Introduction}

Hemophilia A is a bleeding disorder caused by a deficiency or dysfunction of coagulation factor VIII (fVIII). Patients affected by the severe form of the disease suffer from spontaneous bleedings, whereas in mild/moderate hemophilia A (fVIII activity 0.01-0.4 $\mathrm{IU} / \mathrm{mL}$ ) bleeding occurs after minor trauma or surgery. Although the alterations of the fVIII molecule leading to severe hemophilia A are well characterized, only scarce information is available about the molecular mechanisms responsible for mild/moderate hemophilia A. ${ }^{1}$

The mature fVIII molecule is a heterodimer of 2332 amino acids, which circulates as a heavy and a light chain noncovalently bound by a divalent metal bridge. ${ }^{2}$ fVIII contains 3 types of domains, the A domains, homologous to ceruloplasmin, a unique $\mathrm{B}$ domain, and $2 \mathrm{C}$ domains, homologous to the phospholipid binding protein discoidin. The heavy chain is made up by the A1 and A2 domains and by variable lengths of the $\mathrm{B}$ domain. The light chain consists of the $\mathrm{A} 3, \mathrm{C} 1$, and $\mathrm{C} 2$ domains. $^{2}$

In plasma, fVIII circulates bound to von Willebrand factor (vWf). The latter interaction is crucial for fVIII stability as indicated by the low fVIII levels observed in patients with severe von Willebrand disease $(\mathrm{vWD})^{3}$ and in patients with vWD type 2 Normandy, with altered binding of vWf to fVIII. ${ }^{4}$
In vitro experiments have established that $\mathrm{vWf}$ is required for stable association of fVIII heavy and light chains, efficient fVIII production, ${ }^{5,6}$ and protection from enzymatic degradation, notably by activated protein $\mathrm{C}$ and factor $\mathrm{Xa}^{7,8} \mathrm{vWf}$ also prevents fVIII binding onto phospholipids. ${ }^{9-11}$ Two fVIII regions were shown to be involved in fVIII binding to vWf: the amino-terminal part of the A3 domain and the $\mathrm{C} 2$ domain. ${ }^{12-18}$

Recent observations ${ }^{19,20}$ have suggested that residues located outside the A3 and C2 domains could also contribute to the binding of fVIII to vWf. Thus, mutation Arg2150His in the C1 domain alters the binding of an inhibitor antibody that recognizes fVIII only in the presence of vWf. Analysis of Arg2150His fVIII binding to $v W f$ indicated that this substitution reduced the binding of fVIII to $v W f$, which prevented the binding of the antibodies recognizing fVIII bound to vWf. ${ }^{20}$ In addition, a human monoclonal antibody to the fVIII C1 domain was shown to inhibit fVIII binding to vWf. ${ }^{21}$ Residues located in the $\mathrm{C} 1$ domain could, therefore, interfere, directly or indirectly, with fVIII binding to vWf. To address this question, we examined the interaction between vWf and fVIII variants carrying a point mutation in the fVIII C1 domain, resulting in mild/moderate hemophilia A.
From the Center for Molecular and Vascular Biology, University of Leuven, Leuven, Belgium; Hôpital Bicêtre, le Kremlin-Bicêtre, AP-HP, France; Institut für Experimentelle Hämatologie und Transfusionsmedizin, Bonn, Germany; and the Center for Transgene Technology and Gene Therapy, Flanders Interuniversity Institute for Biotechnology, University of Leuven, Leuven, Belgium.

Submitted September 9, 1999; accepted March 23, 2000.

Supported in part by research grant G.0292.98 from the Flemish Research Foundation and by grant Schw 752/1-1 from the Deutsche Forschungsgemeinschaft.

\section{J.V. is holder of the Dr Jean Choay Chair for Hemostasis Research.}

Reprints: Marc Jacquemin, Center for Molecular and Vascular Biology, University of Leuven, Campus Gasthuisberg, O\&N, Herestraat 49, B-3000 Leuven, Belgium; e-mail: marc.jacquemin@med.kuleuven.ac.be.

The publication costs of this article were defrayed in part by page charge payment. Therefore, and solely to indicate this fact, this article is hereby marked "advertisement" in accordance with 18 U.S.C. section 1734.

(C) 2000 by The American Society of Hematology 


\section{Patients, materials, and methods}

\section{Reagents and buffers}

Full-length human recombinant (r)fVIII (specific activity of $4000 \mathrm{IU} / \mathrm{mg}$ ) was generously provided by Hyland (Glendale, CA). Ortho-phenylenediamine, phenylmethylsulfonyl fluoride (PMSF), and avidin-peroxidase were purchased from Sigma Chemical Co (St. Louis, MO). Coatest Factor VIII was bought from Chromogenix AB (Mölndal, Sweden) and Factor VIII Chromogenic Assay from Dade (Düdingen, Switzerland).

\section{Patients}

Plasma was obtained from normal donors, from patients with mild hemophilia A, and from a patient with vWD type 2 with compound heterozygosity for a substitution Arg91Gln on one allele and very low levels of messenger RNA (mRNA) from the second vWf allele. ${ }^{22} \mathrm{~A}$ full clinical description of patient LE has been given elsewhere. ${ }^{19}$ Institutionally approved informed consent was obtained from all patients and normal subjects.

\section{Protein preparation}

vWf was purified by gel filtration chromatography in the presence of 0.5 $\mathrm{mol} / \mathrm{L} \mathrm{CaCl} \mathrm{C}_{2}$ from commercially available Factor VIII vWf SD (Belgian Red Cross) as previously described. ${ }^{21} \mathrm{rfVIII}$ produced by stably transfected Chinese hamster ovary (CHO) cell lines incubated in conditioned medium devoid of vWf and fetal calf serum (FCS) was partially purified by ion exchange chromatography. Conditioned medium was applied on a Mono Q Fast Flow column (Pharmacia) and washed with $20 \mathrm{mmol} / \mathrm{L}$ HEPES, 150 $\mathrm{mmol} / \mathrm{L} \mathrm{NaCl}, \mathrm{CaCl}_{2} 5 \mathrm{mmol} / \mathrm{L}, \mathrm{pH} 7.2$ (HEPES buffer). The proteins were eluted with HEPES buffer, supplemented with $1 \mathrm{~mol} / \mathrm{L} \mathrm{NaCl}$ and $0.1 \%$ Tween-80, stabilized by addition of $0.1 \%$ bovine serum albumin (BSA), concentrated using solid polyethylene glycol, dialyzed against HEPES buffer, and stored at $-80^{\circ} \mathrm{C}$.

\section{Measurement of fVIII binding to vWf in plasma}

fVIII binding to vWf in plasma was evaluated by using a centrifugation assay. ${ }^{20}$ Thus, the vWf-specific moab75H4B12 immunoglobulin M (IgM) antibody was coupled to CNBr-activated Sepharose-4B beads (Pharmacia Biotech), according to the manufacturer's instruction. Plasma samples were diluted 10 -fold in Tris $50 \mathrm{mmol} / \mathrm{L}, \mathrm{NaCl} 0.15 \mathrm{~mol} / \mathrm{L}$, BSA $1 \%$, Tween- 80 $0.1 \%, \mathrm{pH} 7.4$ (TBS-BSA); $50 \mu \mathrm{L}$ of each diluted plasma were mixed with $50 \mu \mathrm{L}$ of the anti-vWf Sepharose (50\% suspension), or of a control Sepharose, for an incubation of 90 minutes at $4^{\circ} \mathrm{C}$. After centrifugation, the amount of fVIII present in the supernatant of the anti-vWf and control Sepharose was evaluated in a chromogenic assay (Coatest Factor VIII), according to the manufacturer's recommendations except that, when required, plasma samples were diluted in TBS-BSA. Initial experiments had established that TBS-BSA was optimal to prevent nonspecific binding of plasma fVIII to Sepharose and could be used to dilute plasma in the Coatest factor VIII. fVIII bound to vWf was calculated by subtracting the amount of fVIII in the supernatant of the anti-vWf Sepharose from that present in the supernatant of the control Sepharose.

To evaluate the binding of rfVIII to vWf in plasma, rfVIII was added at a final concentration of $0.1 \mathrm{IU} / \mathrm{mL}$ to the plasma of a patient with severe hemophilia A. After 30 minutes of incubation at $20^{\circ} \mathrm{C}$, the fraction of fVIII bound to vWf was measured as above.

\section{Scatchard analysis of rfVIII binding to vWf}

Sepharose beads coated with vWf were prepared by mixing $200 \mu \mathrm{L}$ of the anti-vWf moab75H4B12 Sepharose (50\% suspension) with $200 \mu \mathrm{L}$ vWf $(1.8,5.5,16.6$, or $50 \mu \mathrm{g} / \mathrm{mL})$ or control buffer for 60 minutes at $20^{\circ} \mathrm{C}$. After centrifugation, the amount of vWf present in the supernatant of the anti-vWf moab75H4B12 Sepharose was evaluated by enzyme-linked immunosorbent assay (ELISA). The fraction of vWf bound to Sepharose was calculated by subtracting the amount of vWf recovered in the supernatant of anti-vWf Sepharose from the total vWf added to the assay.
After 5 washes in TBS-BSA, vWf Sepharose was resuspended in $800 \mu \mathrm{L}$ TBS-BSA.

rfVIII $(50 \mu \mathrm{L})$ at 0.05 to $5.5 \mathrm{nmol} / \mathrm{L}$ was incubated for 90 minutes at $20^{\circ} \mathrm{C}$ with $50 \mu \mathrm{L}$ vWf Sepharose or $50 \mu \mathrm{L}$ control Sepharose. After centrifugation for 10 seconds at $10000 \mathrm{~g}$, total and free fVIII were measured by a chromogenic assay in the supernatants of control and vWf Sepharose, respectively. Bound fVIII was calculated by subtracting free fVIII from total fVIII. Initial experiments with normal rfVIII established that fVIII binding was evaluable with the use of a fVIII chromogenic assay provided that bound fVIII was greater than $5 \%$ and less than $95 \%$ of total fVIII.

\section{Immunoassays}

Plasma fVIII antigen levels were measured by ELISA with the use of the Immunozym FVIII:Ag test (Immuno AG, Vienna, Austria) following the manufacturer's recommendations.

rfVIII levels were measured by ELISA, according to published methods, ${ }^{19}$ using moab $\mathrm{F} 4 \mathrm{H} 12$ or F15B12, recognizing the $\mathrm{A} 1$ or $\mathrm{A} 2$ domain, respectively, as capture antibodies. Bound fVIII was detected by the addition of a mixture of moab 13, F8D6, F29A1, and F14A12. ${ }^{19} \mathrm{vWf}$ levels were measured by ELISA, according to published methods. ${ }^{21}$

\section{Plasmid mutagenesis}

Mutagenesis was performed within the mammalian expression vector pGCSamF8EN ${ }^{23}$ (provided by R.A. Morgan) coding for the B domain-less fVIII complementary DNA (cDNA). Mutant plasmids were generated through oligonucleotide site-directed mutagenesis, utilizing the polymerase chain reaction. ${ }^{24}$ Codon $\mathrm{n}^{\circ} 2098$ was mutated from ATC to AGC, predicting an amino acid change from I to S. Codon n ${ }^{\circ} 2119$ was mutated from TCC to TAC, predicting an amino acid change from $\mathrm{S}$ to $\mathrm{Y}$. Codon $\mathrm{n}^{\circ} 2150$ was mutated from CGT to CAT, predicting an $\mathrm{R}$ to $\mathrm{H}$ amino acid change. All mutated cDNAs were controlled by sequencing in both directions, using an ABI (Genetic Analyser 3.10 PerkinElmer).

\section{CHO cell transfection}

To establish cell lines expressing wild-type or mutant fVIII, CHO cells were transfected with pGCSamF8EN or the mutated vector by using FUGENE 6 (Boehringer Mannheim, Brussels, Belgium), according to the manufacturer's instructions. The cell lines producing the highest fVIII amounts were expanded and subcloned twice.

\section{FVIII production by transiently transfected $\mathrm{CHO}$ cells}

Transient transfections were performed in a similar manner. $\mathrm{CHO}$ cells $\left(8 \times 10^{4}\right.$ cells/well) were seeded in 6-well plates (Life Technologies), using MEMAlpha Medium (MEM $\alpha$; Life Technologies Ltd, Paisley, UK) supplemented with $10 \%$ FCS (MEM $\alpha$-FCS). After 24 hours of incubation, a transfection mixture of $0.5 \mu \mathrm{g}$ DNA in $10 \mu \mathrm{L}$ of Tris-EDTA, $100 \mu \mathrm{L}$ OPTIMEM, and $2 \mu \mathrm{L}$ FUGENE 6 was applied to the cells. After 48 hours, the cells were washed twice with MEM $\alpha$, and the culture medium was replaced by MEM $\alpha$ supplemented with Nutridoma-CS (Boehringer Mannheim, Germany) and $3 \mathrm{mmol} / \mathrm{L}$ sodium butyrate (MEM $\alpha$-Nutridoma). After 16 hours, the conditioned medium was harvested, centrifuged to remove cell debris, and assayed for fVIII activity. This conditioned medium was used because preliminary experiments had shown that higher fVIII levels could thus be obtained in the absence of serum and of vWf. Seventy-two hours after transfection, cells incubated in MEM $\alpha$-Nutridoma were washed in methionine-free MEM $\alpha$ and incubated for 30 minutes in methionine-free medium containing $0.5 \mathrm{mCi} / \mathrm{mL}$ of $\left[{ }^{35} \mathrm{~S}\right]$ methionine (Amersham) and aprotinin (0.5\%; Sigma). ${ }^{5}$ Intracellular levels of fVIII translation products were evaluated by immunoprecipitation, followed by SDS-PAGE and autoradiography. ${ }^{25}$

After 30 minutes of incubation, the cells were harvested and lysed in $150 \mathrm{mmol} / \mathrm{L} \mathrm{NaCl}, 50 \mathrm{mmol} / \mathrm{L}$ Tris, $\mathrm{pH} 7.4,0.05 \%$ (w/v) SDS, $1 \%$ (v/v) $\mathrm{NP}-40,1 \mathrm{mmol} / \mathrm{L}$ PMSF, and $1 \mathrm{mg} / \mathrm{mL}$ soybean trypsin inhibitor (Tris-NP40). Equal volumes of cell extracts were incubated with $500 \mu \mathrm{L}$ human antibody at $2 \mu \mathrm{g} / \mathrm{mL}$ in Tris-NP-40 supplemented with $0.25 \%$ gelatin and $5 \%$ BSA (Tris-NP-40-Gel-BSA). The tubes were gently rocked for 2 hours at $4{ }^{\circ} \mathrm{C}$. A $50 \%$ solution of Protein A Sepharose $(20 \mu \mathrm{L})$ was then added to 
the antigen/antibody mixture and incubated for 1 hour at $4^{\circ} \mathrm{C}$ on a rocking platform. Sepharose beads were centrifuged and washed twice with Tris-NP-40-Gel-BSA and once in $10 \mathrm{mmol} / \mathrm{L}$ Tris, $\mathrm{pH} 7.5,1 \% \mathrm{v} / \mathrm{v}$ NP40. Bound antigen/antibody complexes were eluted from the beads by boiling for 4 minutes in $30 \mu \mathrm{L}$ of SDS gel loading buffer. An aliquot of $25 \mu \mathrm{L}$ was analyzed by $8 \%(\mathrm{w} / \mathrm{v})$ PAGE and visualized by autoradiography.

The relative concentrations of the fVIII primary translation products were evaluated by comparison with serial dilutions of immunoprecipitates from a reference $\left[{ }^{35} \mathrm{~S}\right]$-labeled cell extract.

\section{mRNA quantification}

Total RNA was isolated from $10^{6}$ transiently transfected $\mathrm{CHO}$ cells by using the TRIzol Reagent (Life Technologies, Gaithersburg, MD). After DNase treatment of the RNA samples, a first strand cDNA synthesis was carried out with the Superscript Preamplification system, according to the instructions of the manufacturer (Life Technologies).

Quantitative real-time PCR was carried out with the use of gene-specific double fluorescently labeled probes in a 7700 Sequence Detector (PE Applied Biosystems, Norwalk, CT). 6-Carboxyfluorescein, FAM, and JOE, were used as the $3^{\prime}$ fluorescent reporter for fVIII and hypoxanthine guanine phosphoribosyl transferase (HPRT), respectively, whereas tetramethylrhodamine (TAMRA) was used as the $5^{\prime}$ quencher. The following primer and probe sequences were used: HPRT forward primer, $5^{\prime}$-TTATCAGACTGAAGAGCTACTGTAATGATC-3'; HPRT reverse primer, $5^{\prime}$-TTACCAGTGTCAATTATATCTTCAACAATC-3'; HPRT probe, $5^{\prime}$-TAMRA-TGAGAGATCATCTCCACCAATAACTTTTATGTCCC-JOE-3'; fVIII forward primer, 5'-AACCGAAGCTGGTACCTCACA-3'; fVIII reverse primer, 5'-GGATCCTCAAGCTGCACTCC-3'; and fVIII probe, 5'-TAMRAAGAATATACAACGCTTTCTCCCCAATCC-FAM-3'. All probes were designed to span exon junctions in the fully processed message to prevent reporting of amplification of any possible contaminating genomic DNA. The cDNA samples underwent a denaturating step of 10 minutes at $95^{\circ} \mathrm{C}$ and 40 cycles of 15 seconds at $95^{\circ} \mathrm{C}$ and 1 minute at $60^{\circ} \mathrm{C}$, yielding an amplicon of 126 and 74 base pairs (bp) for HPRT and fVIII, respectively.

\section{fVIII immunoprecipitation and immunoblot analysis}

Normal and mutant fVIII (1 IU) were immunoprecipitated with the human monoclonal antibody $\mathrm{BO} 2 \mathrm{C} 11,{ }^{26}$ according to published methods. Immunoprecipitates were analyzed by Western blotting with the use of moab12 and F14A12.

\section{fVIII production in presence and absence of vWf}

To evaluate the stabilization of fVIII by vWf, cell cultures were performed, according to published methods. ${ }^{5}$ Stably transfected $\mathrm{CHO}$ cells were seeded at $5 \times 10^{4}$ per well in 48 -well plates and incubated overnight at $37^{\circ} \mathrm{C}$ in MEM $\alpha$ supplemented with $10 \%$ FCS. The cells were then washed 3 times and the cell culture medium was replaced by Opti-MEM (Life Technologies) with $3 \mathrm{mmol} / \mathrm{L}$ sodium butyrate, supplemented or not with various amounts of vWf. After 16 hours at $37^{\circ} \mathrm{C}$, the cell culture supernatants were harvested and assayed for fVIII activity.

\section{Statistical analysis}

The significance of the differences was determined by using the Student $t$ test for unpaired values.

\section{Results}

\section{Binding of plasma fVIII to vWf}

Although mutations in the fVIII C1 domain are commonly found in patients with mild/moderate hemophilia, ${ }^{1}$ no role(s) has yet been ascribed to the $\mathrm{C} 1$ domain in fVIII synthesis, secretion, stability, or cofactor function. To evaluate whether mutations in the $\mathrm{C} 1$ domain resulted in a reduction of fVIII binding to vWf, plasmas of patients with mild/moderate hemophilia A with such mutations were analyzed. Tenfold diluted plasmas were incubated with Sepharose coupled to an anti-vWf antibody. At equilibrium, the Sepharose was centrifuged, and unbound vWf and fVIII were measured. In all experiments, more than $95 \%$ vWf was captured on the Sepharose. For 7 normal plasmas and a pool of normal plasmas, the fraction of bound fVIII was comprised between $71.1 \%$ and $80.9 \%(\mathrm{X}=73.1$, $\mathrm{SD}=3.7$ ) (Figure 1A). To rule out an effect of the fVIII:c plasma level in the assay system, the plasma of a normal donor was diluted 10- or 50-fold in the plasma of a patient with severe hemophilia A. The fraction of fVIII bound to vWf was $76 \% \pm 3 \%$ and $81 \% \pm 2 \%$, respectively (ie, within the normal range of the assay). In a further control experiment, the plasma of a patient with vWD type 2 Normandy showed strongly reduced fVIII binding in the assay system $(35 \% \pm 11 \%)$.

fVIII with mutations Ile312Val, Gly479Arg, Leu625Val, and Asn2019Ser in the A1, A2, and A3 domain, respectively, bound to vWf to the same extent as normal fVIII (Figure $1 \mathrm{~B}$ and C). As shown in Figure 1D, of 7 variants with mutations in the $\mathrm{C} 1$ domain resulting in mild/moderate hemophilia A, only 2 showed a normal binding to vWf. The substitutions Ile2098Ser, Ser2119Tyr, Asn2129Ser, Arg2150His, and Pro2153Gln in the C1 domain were characterized by a smaller fraction of bound fVIII (range 13\%-67\%).

For the 5 mutations associated with reduced fVIII/vWf binding, the levels of fVIII:Ag and fVIII:c were closely related, indicating that the specific activity of the mutated fVIII molecules was close to that of normal fVIII (Table 1). Hence, the mechanisms responsible for reduced fVIII levels in the plasma of these patients were likely to involve either reduced synthesis and/or secretion or reduced stability in plasma. These questions were addressed with the use of rfVIII variants produced by $\mathrm{CHO}$ cells transfected with cDNA expression vectors for B domain-deleted fVIII.

\section{Binding of lle2098Ser, Ser2119Tyr, Arg2150His rfVIII mutants to vWf}

Three representative fVIII mutants were selected for these experiments: Ser2119Tyr (ie, the mutant with the lowest binding to $\mathrm{vWf}$ ), Ile2098Ser, and Arg2150His. An expression vector coding for a B
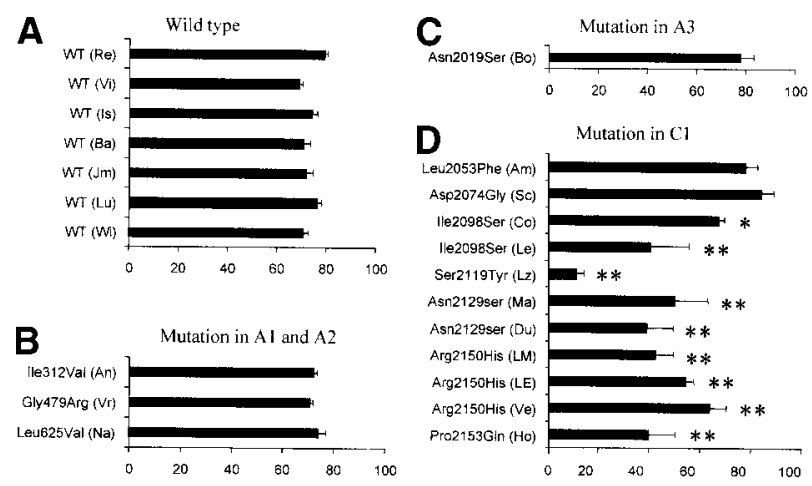

Figure 1. Binding of plasma fVIII to vWf. Sepharose beads coated with anti-vWf moab75H4B12 were incubated with 10-fold diluted plasma. The beads were centrifuged, and fVIII present in supernatants was measured in a chromogenic assay. Control experiments were carried out with uncoated Sepharose beads. Results are expressed as the percentage of fVIII bound to $\mathrm{VWf}$ (Bound $\mathrm{fVIII}(\%)=[\mathrm{fVIII}$ in the supernatant of control Sepharose - fVIII in the supernatant of anti-VWF Sepharose] $\times 100 / f V I I I$ in the supernatant of control Sepharose). (A) Normal donor plasma fVIII; (B) plasma of patients with mild/moderate hemophilia A with mutations in the fVIII A1 or A2 domain; (C) plasma of a patient with mild/moderate hemophilia A with mutation in the fVIII A3 domain; (D) plasmas with fVIII mutations in the C1 domain. Each value represents the mean of at least 2 experiments and SDs are as indicated. Values of vWf-bound fVIII significantly lower than that of normal fVIII are indicated: ${ }^{*} P<.025 ;{ }^{* *} P<.01$. 
Table 1. fVIII:c and fVIII:Ag of plasma fVIII with reduced binding to vWf

\begin{tabular}{lcc}
\hline \multicolumn{1}{c}{ Mutation } & fVIII: c $^{*}$ & fVIII:Ag† \\
\hline Ile2098Ser (Le) & 4 & 5 \\
Ile2098Ser (Co) & 8 & $\mathrm{ND}$ \\
Ser2119Tyr (ZI) & 4 & $<5$ \\
Asn2129Ser (Ma) & 18 & 12 \\
Arg2150His (Ve) & 3 & $\mathrm{ND}$ \\
Arg2150His (LE) & 9 & $\mathrm{ND}$ \\
Arg2150His (LM) & 9 & 11 \\
Pro2153GIn (Or) & 2 & $<5$ \\
\hline
\end{tabular}

* $\mathrm{f}$ III cofactor activity in patient plasma was measured in a coagulation assay. Results are expressed in percentage of the fVIII cofactor activity of a pool of normal plasma.

ffVIII antigen measured in enzyme-linked immunosorbent assay. Results are expressed in percentage of the fVIII antigen level of a pool of normal plasma. ND indicates no data.

domain-deleted fVIII was selected, because it allowed for a higher fVIII production than a vector encoding the complete fVIII gene. ${ }^{23}$

The affinity of rfVIII variants for $\mathrm{vWf}$ was compared by Scatchard analysis. Initial experiments with normal rfVIII established that fVIII levels evaluable with the use of a fVIII chromogenic assay were obtained with concentrations of $\mathrm{vWf}$ ranging from 0.2 to $6 \mathrm{nmol} / \mathrm{L}$. By contrast, with the mutant fVIII preparations, valid data were obtained only when using a concentration of vWf of $6 \mathrm{nmol} / \mathrm{L}$.

The apparent dissociation constant $\left(\mathrm{K}_{\mathrm{D}}\right)$ of the binding of normal $\mathrm{rfVIII}$ to $\mathrm{vWf}$ was $1.0 \pm 0.3 \mathrm{nmol} / \mathrm{L}$ (Table 2), as determined from binding experiments with the use of different concentrations of vWf insolubilized on Sepharose (Figure 2A). This determination is in agreement with published values $\left(\mathrm{K}_{\mathrm{D}}=0.49 \pm 0.12\right.$ $\mathrm{nmol} / \mathrm{L}) .{ }^{27}$ The calculated number of fVIII binding sites per vWf monomer was 0.4 . Such a low ratio could be attributed to either degraded molecules in the vWf preparation or to limited accessibility of fVIII molecules for insolubilized vWf. The latter hypothesis is more likely, because similar results were obtained by using different vWf preparations (plasma-derived or recombinant vWf preparations, data not shown).

Analysis of the binding of Arg2150His to insolubilized vWf (6 $\mathrm{nmol} / \mathrm{L}$ ) indicated that the dissociation $\mathrm{K}_{\mathrm{D}}$ was 3.3-fold higher than that of normal fVIII. The binding stoichiometry at saturation was similar to that determined for normal fVIII ( $0.4 \mathrm{fVIII}$ molecule per vWf monomer). Under the same experimental conditions, the

Table 2. Binding to vWf and specific activity of normal and mutated rfVIII

\begin{tabular}{lccc}
\hline fVII variant & $\mathrm{K}_{\mathrm{D}}(\mathrm{nM})^{*}$ & $\begin{array}{c}\text { Binding to vWf } \\
\text { in plasma }(\%) \dagger\end{array}$ & $\begin{array}{c}\text { fVIII specific } \\
\text { activity }\end{array}$ \\
\hline Normal & $1.0 \pm 0.3$ & $79 \pm 1$ & $0.6 \pm 0.1$ \\
Ser2119Tyr & $83.3 \pm 28.4$ & $5 \pm 2$ & $0.9 \pm 0.2$ \\
Ile2098Ser & $7.8 \pm 3.2$ & $43 \pm 6$ & $1.1 \pm 0.5$ \\
Arg2150His & $3.3 \pm 1.2$ & $56 \pm 3$ & $0.8 \pm 0.4$ \\
\hline
\end{tabular}

*The dissociation constants $\left(\mathrm{K}_{\mathrm{D}}\right)$ of factor VIII (fVIII) binding to von Willebrand factor (vWf) were evaluated by Scatchard analysis of fVIII binding to vWf Sepharose. Data are presented as mean \pm SD of at least 3 independent experiments.

†Recombinant $\mathrm{fVIII}(0.2 \mathrm{IU} / \mathrm{mL})$ was incubated for 1 hour at $20^{\circ} \mathrm{C}$ in the plasma of a patient with severe hemophilia $A$. The samples were diluted 10 -fold, and the fraction of fVIII bound to vWf was determined, using a Sepharose coated with an anti-vWf mouse monoclonal antibody, as in Figure 1. Results are expressed as the mean \pm $\mathrm{SD}$ of triplicate experiments.

$\ddagger$ fVIII specific activity was evaluated as the ratio between fVIII:C and fVIII:Ag. A chromogenic assay was used to measure fVIII:c. The levels of fVIII:Ag were measured in 3 enzyme-linked immunosorbent assays (ELISAs), using 3 different capture antibodies. For each fVIII variant, 3 evaluations of specific activity were made by determining the ratio between $\mathrm{fVIII:C}$ and $\mathrm{fVIII}: A g$ levels as determined in ELISA. Results are expressed as the mean \pm SD of specific activity determined in the 3 assays.
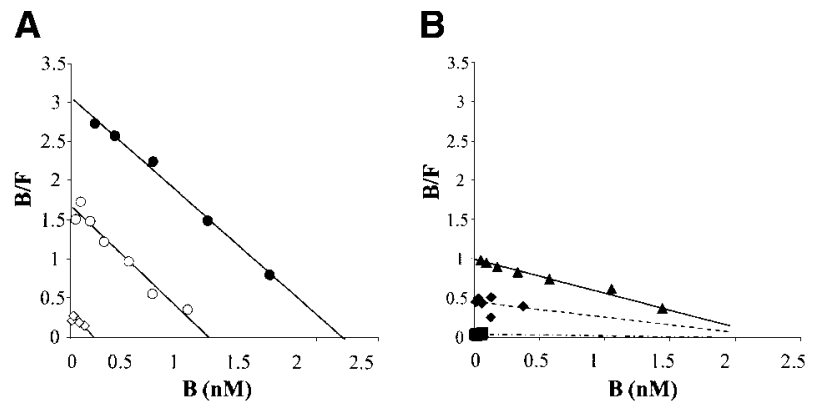

Figure 2. Scatchard analysis of normal and mutant rfVIII binding to vWf. (A) Normal rfVIII $(0.05$ to $5.5 \mathrm{nmol} / \mathrm{L})$ was incubated for 90 minutes at room temperature with vWf insolubilized on Sepharose $(\diamond, 0.2 \mathrm{nmol} / \mathrm{L} ; \bigcirc, 2.4 \mathrm{nmol} / \mathrm{L} ; \bullet$, $5.9 \mathrm{nmol} / \mathrm{L}$ ) or with control Sepharose. After centrifugation, free $(F)$ and total $(T)$ fVIII were measured by a chromogenic assay in the supernatant of control and vWf Sepharose, respectively. Bound (B) fVIII was calculated by subtracting free from total fVIII. The concentration of fVIII bound to $\mathrm{vWf}(\mathrm{B})$ is plotted against the ratio of bound/free (B/F) fVIII. Plain lines represent linear regression analysis of experimental data points. One experiment representative of 3 separate evaluations is shown. (B) Mutant rfVIIls ( $\bullet$ Ile2098Ser; $\mathbf{\square}$, Ser2119Tyr; $\mathbf{\Delta}$, Arg2150His) were incubated at various concentrations $(0.05-5.3 \mathrm{nmol} / \mathrm{L})$ with $v W f-b o u n d ~ S e p h a r o s e ~(5.9 \mathrm{nmol} / \mathrm{L})$. The concentration of fVIII bound to $\mathrm{VWf}(\mathrm{B})$ is plotted against the ratio of bound/free $(B / F)$ fVIII. Plain lines represent linear regression analysis of experimental data points. Dotted lines represent the linear regression analysis of experimental data points and of an arbitrary point, corresponding to the maximal number of fVIII binding sites on vWf. This number was calculated as the intersection with the $x$ axis of the line corresponding to the linear regression analysis of experimental data points of normal and Arg2150His rfVIII binding to vWf. One experiment representative of 3 separate evaluations is shown.

binding of Ile2098Ser or Ser2119Tyr fVIII to vWf was too low to provide enough data points to calculate the number of binding sites at saturation and the $K_{D}$ of the reaction. For these 2 mutants, the $K_{D}$ was, therefore, determined by taking into account an arbitrary point corresponding to the number of fVIII binding sites present on insolubilized vWf. This number was derived from the Scatchard analyses of the binding of normal and Arg2150His fVIII to vWf (Figure 2A-B). By using this method, the calculated $\mathrm{K}_{\mathrm{D}}$ of Ser2119Tyr and Ile2098Ser fVIII binding to vWf was about 80 -fold and 8-fold higher than that of normal fVIII, respectively (Table 2), in agreement with the reduced $\mathrm{fVIII} / \mathrm{vWf}$ interaction observed in plasma containing these mutants (Figure 1).

To evaluate the vWf-binding capacity of rfVIII molecules in plasma, a fixed concentration of fVIII:c $(0.1 \mathrm{IU} / \mathrm{mL})$ was added to the plasma of a patient with severe hemophilia A. After 30 minutes of incubation at $20^{\circ} \mathrm{C}$, the plasmas were diluted 10 -fold and the fraction of fVIII bound to $\mathrm{vWf}$ was determined after incubation with a Sepharose coated with a murine anti-vWf monoclonal antibody. As shown in Table 2, the binding to vWf of normal rfVIII was in accordance with the binding of normal plasma fVIII when assayed under similar conditions (Figure 1).

The fractions of mutated rfVIII molecules bound to vWf were significantly reduced by comparison to normal rfVIII (Table 2) and were within the same range of magnitude as the fractions of mutant fVIII molecules associated to vWf in patients' plasma (Figure 1). Thus, for recombinant and plasma fVIII, the lowest association with vWf was observed with Ser2119Tyr mutant, in close agreement with the low affinity for vWf determined for this mutant by Scatchard analysis (Table 2).

\section{Subunit composition and specific activity of mutant rfVIII molecules}

The reduced binding to vWf could still be due to an alteration of the mutant rfVIII molecules. Therefore, the physical state of the fVIII molecules was analyzed by Western blot, and the specific activity 


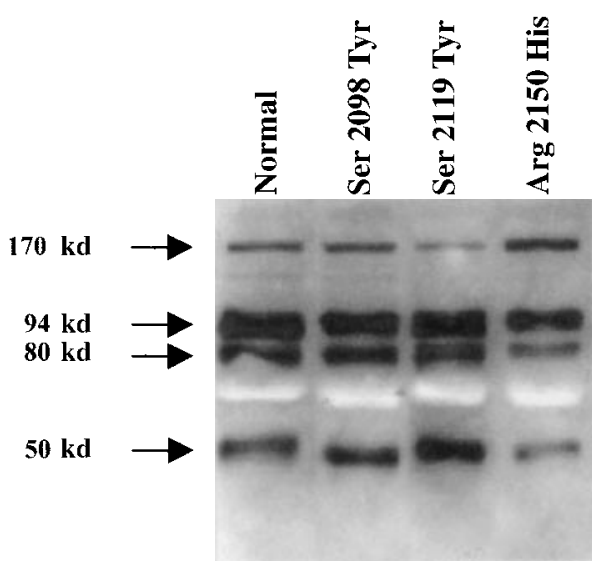

Figure 3. Western blot analysis of recombinant normal and purified fVIII. Partially purified normal and mutant rfVIII molecules were immunoprecipitated by using the human monoclonal antibody $\mathrm{BO} 2 \mathrm{C} 11$ bound on protein $\mathrm{A}$ Sepharose and analyzed by SDS-PAGE followed by Western blotting. Mouse anti-fVIII moab 12 and F14A12 were used to detect fVIII fragments.

of fVIII mutants was determined with the use of a fVIII functional assay and ELISA.

The subunit composition of purified rfVIII was analyzed by Western blotting analysis. To eliminate BSA present in the fVIII preparations, and which altered the migration of the fVIII chains on SDS-PAGE, fVIII molecules were immunoprecipitated before analysis. To carry out the precipitation, the human monoclonal antibody BO2C11 was selected for its high affinity for $\mathrm{fVIII}^{26}$ and because of its ability to completely inhibit the functional activity of the $3 \mathrm{fVIII}$ mutants, which was taken as a further indication that the mutations had not altered the epitope recognized by the antibody.

The amounts of fVIII light chain were similar in preparations of normal and mutant molecules when identical amounts of fVIII were used as adjusted by functional activity (Figure 3 ). This finding excluded that isolated light chains able to bind to vWf that could have interfered within the binding experiments reported above. The amounts of heavy chain were also similar for the $4 \mathrm{rfVIII}$, indicating that the proportion of light chains associated to heavy chains were similar in normal and mutant proteins. This finding strongly suggested that there was no difference between normal and mutant fVIII in the cleavage of the single-chain form of fVIII. All fVIII preparations also contained an additional fragment with a molecular weight of $50 \mathrm{kd}$. The amounts of the latter were similar in all preparations of normal and mutated fVIII (Figure 3).

To evaluate the specific activity of the rfVIII molecules, fVIII levels measured in chromogenic assays were compared with fVIII antigen levels determined in ELISA. To avoid a bias because of mutation-induced alteration of epitopes or because of degradation fragments, fVIII antigen levels were evaluated in 3 different ELISAs, using capture antibodies recognizing either the light chain, the A1 domain, or the A2 domain, respectively. Concordant results were obtained by the 3 assays, and the specific activities (fVIII:c/fVIII:Ag) of the 3 recombinant molecules were close to that of normal rfVIII (Table 2) and of their plasma counterparts (Table 1). Taken together, these data excluded that alterations of the rfVIII molecules could have introduced a bias in the evaluation of the vWf binding capacity of mutant rfVIII molecules.

\section{In vitro production of fVIII lle2098Ser, Ser2119Tyr, and Arg2150His fVIII}

A reduction of fVIII secretion is an important physiopathological mechanism responsible for hemophilia A. Two other laboratories $^{28,29}$ demonstrated that mutations responsible for mild/moderate hemophilia A and located at residue Arg2307 in the fVIII C2 domain strongly reduced the fVIII secretion rate. We, therefore, determined whether, in addition to reduction of fVIII binding to vWf the mutations Ile2098Ser, Ser2119Tyr, and Arg2150His could also result in a reduced fVIII synthesis and/or secretion.

The experiments were performed by using $\mathrm{CHO}$ cells transiently transfected with expression vectors for normal or mutated $\mathrm{B}$ domain-deleted fVIII. In all experiments, $\mathrm{CHO}$ cells were washed 48 hours posttransfection and incubated in conditioned medium devoid of vWf.

The fVIII mRNA steady state levels were evaluated by real-time quantitative PCR in $\mathrm{CHO}$ cell extracts 64 hours after transfection. As shown in Table 3, the fVIII mRNA levels were similar in cells transfected with cDNA expression vectors for Ser2119Tyr, Ile2098, Arg2150His, and normal fVIII.

The efficiency of fVIII translation was evaluated in duplicate cell cultures, by short labeling with $\left.{ }^{35} \mathrm{~S}\right]$ methionine and immunoprecipitation of total cell extracts with a pool of anti-fVIII antibodies. Intracellular mutants were detected as a single-chain form, migrating at approximately $170 \mathrm{kd}$, as for normal fVIII (Figure 4). The intensity of the bands corresponding to the 3 mutants and normal fVIII varied slightly (Figure 4). However, there was no consistent effect of these mutations on the rate of fVIII protein translation when compared with the corresponding fVIII mRNA steady-state levels (Table 3).

The rate of functional fVIII secretion by transiently transfected $\mathrm{CHO}$ cells was assessed by measuring fVIII:c activity accumulated in conditioned medium devoid of vWf during the 16 hours preceding $\left[{ }^{35} \mathrm{~S}\right]$ methionine labeling or RNA extraction. Relative to the fVIII primary translation products, the rate of accumulation of Ser2119Tyr was equal to that of normal fVIII (Table 3), whereas

Table 3. mRNA steady-state levels, translation, and secretion of normal and mutated fVIII by transiently transfected CHO cells

\begin{tabular}{|c|c|c|c|c|c|}
\hline fVIII Gene & $\mathrm{mRNA}^{*}$ & Translation† & Translation/mRNA & Secretion $\ddagger$ & Secretion/translation \\
\hline Normal & $1.0 \pm 0.1$ & $1.0 \pm 0.3$ & $1.0 \pm 0.2$ & $1.0 \pm 0.1$ & $1.0 \pm 0.1$ \\
\hline Ser2119Tyr & $0.7 \pm 0.2$ & $0.9 \pm 0.5$ & $0.9 \pm 0.7$ & $1.3 \pm 0.2$ & $1.6 \pm 0.6$ \\
\hline Ile2098Ser & $0.7 \pm 0.12$ & $0.7 \pm 0.3$ & $0.7 \pm 0.3$ & $0.4 \pm 0.1$ & $0.6 \pm 0.2$ \\
\hline Arg2150His & $0.7 \pm 0.1$ & $0.9 \pm 0.2$ & $0.9 \pm 0.3$ & $0.14 \pm 0.03$ & $0.16 \pm 0.06$ \\
\hline
\end{tabular}

${ }^{*}$ Factor VIII (fVIII) and hypoxanthine guanine phosphoribosyl transferase (HPRT) messenger RNA (mRNA) steady-state levels were determined by quantitative real-time PCR of total mRNA extracted from Chinese hamster ovary $(\mathrm{CHO})$ cells 72 hours after transfection with an expression vector for mutant or normal fVIII. After correction for HPRT levels, fVIII mRNA levels were expressed relative to fVIII mRNA levels in CHO cells transfected with the expression vector for normal fVIII.

†fVIII translation efficiency was determined by a short [ $\left.{ }^{35} \mathrm{~S}\right]$ methionine labeling of $\mathrm{CHO}$ cells 72 hours after transfection with expression vector for mutant or normal fVIII. Total cell lysates were analyzed by immunoprecipitation with monoclonal antibodies directed toward fVIII light and heavy chains, followed by SDS-PAGE and autoradiography. Results are expressed relative to the levels of fVIII single-chain primary translation product in $\mathrm{CHO}$ cells transiently transfected with the expression vector for normal fVIII.

¥fVIII secretion was assessed by measuring fVIII:C activity accumulated in conditioned medium of $\mathrm{CHO}$ cells during 12 hours preceding [ $\left.{ }^{35} \mathrm{~S}\right] \mathrm{methionine} \mathrm{labeling} \mathrm{or} \mathrm{RNA}$ extraction. Results are expressed relative to fVIII:C activity in the conditioned medium of CHO cells transfected with an expression vector for normal fVIII. Mean \pm SD of 2 experiments are shown. 


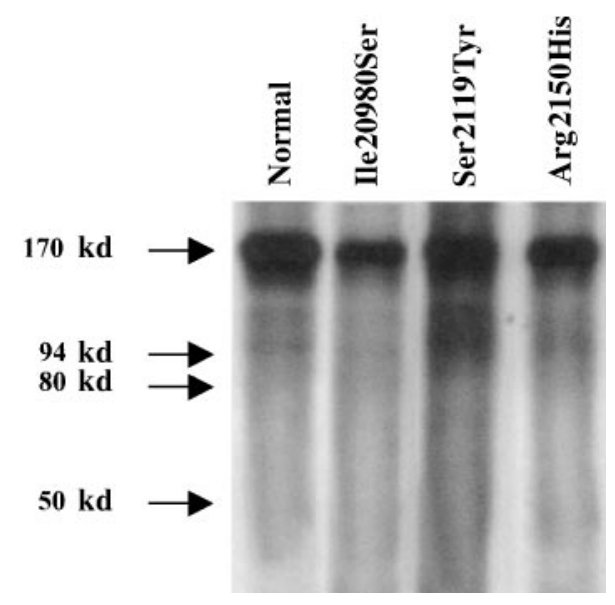

Figure 4. Normal and mutant fVIII translation efficiencies. $\mathrm{CHO}$ cells transiently transfected with normal and mutant fVIII cDNA expression vectors were labeled with $\left[{ }^{35} \mathrm{~S}\right] \mathrm{methionine}$ for 30 minutes. Cell extracts were analyzed by immunoprecipitation with a pool of monoclonal antibodies to fVIII light and heavy chains. Molecular weight markers are shown on the left. One experiment representative of 3 separate evaluations is shown.

those of Ile2098Ser and Arg2150His were reduced about twofold and sixfold, respectively. In control experiments, normal or mutant rfVIII were incubated at $37^{\circ} \mathrm{C}$ for various periods of time in the presence of $\mathrm{CHO}$ cells, and the kinetics of disappearance of fVIII from the conditioned culture medium were evaluated by using a fVIII chromogenic assay. The half-times of disappearance of mutant and normal fVIII were similar (data not shown), excluding that differences in the stability and/or rate of clearance between normal and mutant rfVIII could significantly affect accumulation of functional $\mathrm{fVIII}$ in the absence of vWf. Accordingly, the rate of accumulation of fVIII in transfected $\mathrm{CHO}$ cell conditioned medium likely reflects the rate of fVIII secretion.

\section{fVIII accumulation in the presence of vWf}

As fVIII/vWf association is crucial for in vivo fVIII stability, it was likely that the alteration of fVIII binding to vWf observed for the 3 mutants characterized above reduced the stability of fVIII in plasma. To determine in vitro whether alteration of fVIII/vWf interaction resulted in a reduction of fVIII production and/or stability, we assessed the accumulation of fVIII in the conditioned medium of stably transfected CHO cell lines producing Ser2119Tyr, Ile2098Ser, and Arg2150His fVIII in the presence of various concentrations of vWf.

As shown in Figure 5, the accumulation of normal fVIII was tripled in the presence of $1 \mu \mathrm{g} / \mathrm{mL} v W f$. The maximal (ninefold) increase of fVIII concentration was achieved with $25 \mu \mathrm{g} / \mathrm{mL}$ vWf. By contrast, the concentration of the 3 mutants was enhanced to a lesser extent by vWf. For Ser2119Tyr, Ile2098Ser, and Arg2150His fVIII a 3-fold fVIII increase was achieved with vWf concentrations 16-fold, 8-fold, and 3-fold higher, respectively, than for normal fVIII (Figure 5). Thus, for the 3 mutants, the deficient accumulation in the presence of vWf closely reflected the reduced fVIII binding to $\mathrm{vWf}$, as evaluated in plasma (Figure 1) or in a purified system (Figure 2B; Table 2).

\section{Discussion}

Although many point mutations located in the $\mathrm{C} 1$ domain result in mild/moderate hemophilia $\mathrm{A}$, the function of the $\mathrm{C} 1$ domain is still completely unknown. The observation that mutations located in $\mathrm{C} 1$ alter the binding of fVIII to vWf is remarkable given that this region of the fVIII molecule is not considered to participate in fVIII binding to $\mathrm{vWf}$.

By antibody inhibition assays and recombinant fragment binding assays, 2 regions of the fVIII molecule have been shown to contribute to binding to vWf: a portion of the A3 domain and the C2 domain. ${ }^{12-18}$ The affinity of the separated A3 and C2 domains was respectively 60 and $200 \mathrm{nmol} / \mathrm{L}$, whereas the affinity of the complete light chain was $2 \mathrm{nmol} / \mathrm{L} .^{18}$

Although the role of $\mathrm{C} 2$ in fVIII binding to $\mathrm{vWf}$ is well established, mutations of the $\mathrm{C} 2$ domain leading to alteration of fVIII binding to vWf have not been identified so far. Early evaluation of Arg2307Gln fVIII suggested that the mutation altered fVIII binding to vWf, ${ }^{30}$ but analysis of the rate of fVIII synthesis showed that the low fVIII levels were due to the retention of mutated fVIII in the endoplasmic reticulum, which prevented efficient secretion. ${ }^{28,29}$

In the A3 domain, the only mutations identified so far that result in reduced fVIII binding to vWf are substitutions of tyrosine residue 1680 , which prevent a sulphation critical for fVIII binding to vWf. ${ }^{31,32}$ A possible reason for the difficulty to detect such mutation(s) may be that, in contrast to the $\mathrm{C} 1$ domain, the $\mathrm{A} 3$ and $\mathrm{C} 2$ domains also mediate interactions of fVIII with either coagulation factors or phospholipids. Hence, mutations in the latter domains may frequently be associated to alteration of fVIII cofactor activity in addition to potential disturbance of fVIII binding to vWf.

After the initial description of a patient with mild hemophilia A with an Arg2150His substitution in the $\mathrm{C} 1$ domain and reduced fVIII binding to $\mathrm{vWf},{ }^{20}$ a systematic screening was undertaken on plasmas of patients with $\mathrm{C} 1$ mutations to determine whether this represented a significant mechanism that could account for the mild hemophilia A phenotype. Analysis of $\mathrm{fVIII} / \mathrm{vWf}$ interactions in such plasmas identified 4 other mutations in addition to Arg2150His (ie, Ile2098Ser, Ser2119Tyr, Asn2129Ser, and Pro2153Gln), which resulted in a qualitatively impaired fVIII binding to vWf.

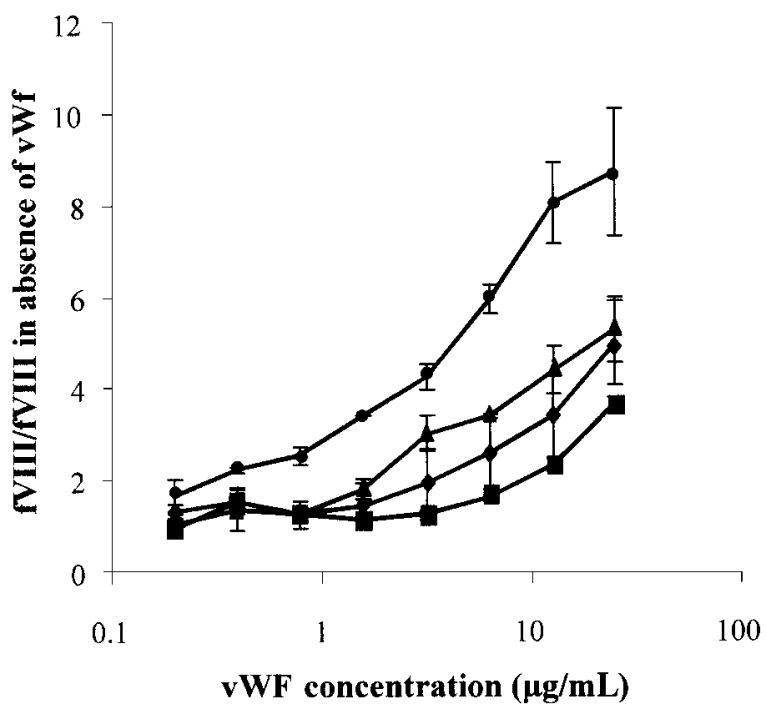

Figure 5. vWf-dependent rfVIII accumulation in conditioned medium of transfected $\mathrm{CHO}$ cells. $\mathrm{CHO}$ cells transfected with expression vectors for normal $(\mathbf{\bullet})$,

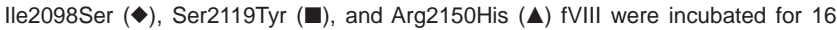
hours in the presence of various concentrations of vWf, as indicated. fVIII activity was determined in a chromogenic assay. Results were expressed as the ratio between $\mathrm{fVIII}: \mathrm{c}$ levels in the presence of $\mathrm{vWf}$ to that in the absence of vWf. One representative experiment of 3 is shown. 
Albeit performed with plasma, the method used for the screening does not allow us to determine the affinity of fVIII binding to $\mathrm{vWf}$ or the fraction of fVIII bound to vWf in plasma in vivo. Indeed, the evaluation of the fraction of vWf-bound fVIII requires the initial capture of $v W f$ on Sepharose coated with anti-vWf monoclonal antibody. To allow for a complete capture of vWf molecules, plasma had to be diluted 10-fold, which displaces the equilibrium toward dissociation of fVIII from vWf. Binding of vWf to Sepharose may also alter the accessibility of some vWf monomers, thereby further displacing the equilibrium toward dissociation of the fVIII/vWf complex. Matrix effect, such as ionic strength, calcium ion concentrations, and detergent, may also theoretically modify fVIII/vWf interaction in comparison to native undiluted plasma.

Other patient-related parameters, such as the concentration of vWf, are likely to significantly influence the evaluation of fVIII binding to vWf in plasma. This is, in fact, illustrated by the observation that, for 3 patients carrying the Arg2150His substitution, the fraction of fVIII bound to vWf varied from $40 \%$ to $67 \%$. We, therefore, analyzed in a purified system the affinity of selected rfVIII mutants for vWf. Scatchard analysis of rfVIII binding to vWf confirmed that the 3 mutants Ile2098Ser, Ser2119Tyr, and Arg2150His had a reduced affinity for vWf. The 80-fold reduced affinity of Ser2119Tyr rfVIII for vWf is in agreement with the severely impaired $\mathrm{fVIII} / \mathrm{vWf}$ interactions observed in the plasma of a patient carrying this mutation, suggesting that, despite all the caveats theoretically associated with the evaluation of fVIII/vWf interactions in plasma, this assay provides results representative of relative affinities of mutated fVIII molecules. Conversely, the modest 3.3-fold reduction of Arg2150His rfVIII affinity for vWf indicates that the analysis of fVIII/vWf complex in plasma is very sensitive for the detection of fVIII variants with reduced binding to vWf.

It is of interest to relate the mutations resulting in reduced fVIII binding to $\mathrm{vWf}$ to the recently proposed a 3-dimensional model of the fVIII $\mathrm{C} 1$ domain. ${ }^{33}$ Four of the 5 mutations occur in residues that are conserved in the discoidin family and are, therefore, more likely to play a structural role in the $\mathrm{C} 1$ domain than to be involved directly in intermolecular interactions with vWf. Ser2119 is located at the interface between the $\mathrm{A} 3$ and $\mathrm{C} 1$ domains, and the substitution to $\mathrm{Y}$ disrupts the domain interface and the global structure of fVIII. Asn2129 is found in a surface loop, whereas Arg2150 and Pro2153 are located in the central $\beta$-sandwich core of the $\mathrm{C} 1$ domain. Mutations of these residues are, therefore, expected to destabilize the $\mathrm{C} 1$ domain and potentially result in misfolded protein. Mutation of Arg2 150 to $\mathrm{H}$ is predicted to be particularly disruptive, ${ }^{33}$ which is a possible explanation for its reduced rate of secretion. The fifth mutation affects Ile 2098, a residue that is not conserved in the discoidin family, although residue hydrophobicity at this location is maintained in the $\mathrm{C} 1$ and $\mathrm{C} 2$ domains of factor $\mathrm{V}$ and in the C2 domain of fVIII. Ile2098 is located in a loop buried in the hydrophobic core of the $\mathrm{C} 1$ domain, and substitution Ile2098 to $\mathrm{S}$ is, therefore, also predicted to result in destabilization and/or impaired folding of the fVIII molecule..$^{34}$

The mechanism by which $\mathrm{C} 1$ influences the affinity of fVIII for $\mathrm{vWf}$ is not yet elucidated. It is possible that the reduction of fVIII binding is due to an impairment of contact between $\mathrm{C} 1$ and $\mathrm{vWf}$ residues. This hypothesis would be compatible with recent observations, showing that a human monoclonal antibody recognizing the C1 domain, LE2E9, is able to completely inhibit fVIII binding to vWf. ${ }^{21} \mathrm{Fab}$ fragments of LE2E9 are also able to interfere with fVIII binding to $\mathrm{vWf}$.

Conversely, mutations in the $\mathrm{C} 1$ domain could have an indirect effect, by altering other regions of the fVIII molecule mediating the contact with vWf, notably within the A3 or C2 domains. The prediction of the structural effects of mutation Ser2119Tyr is compatible with such a phenomenon because of its peculiar location at the $\mathrm{A} 3-\mathrm{C} 1$ interface. ${ }^{33}$

Given the importance of vWf for fVIII stability in plasma, the reduced binding of mutated fVIII to vWf could be responsible for the reduced fVIII levels found in the plasma of the patients. Alternatively, the synthesis/secretion rate of fVIII could be altered. Analysis of fVIII production in the absence of vWf by transiently transfected $\mathrm{CHO}$ cells indicated that the accumulation of functional Ile2098Ser and Arg2150His fVIII molecules was reduced about 2-fold and 6-fold, respectively, by comparison to normal and Ser2119Tyr fVIII. Because, in the absence of vWf, mutant and normal fVIII had a similar stability in the presence of native $\mathrm{CHO}$ cells, the reduced rates of accumulation of Ile2098Ser and Arg2150His fVIII in transfected CHO cells conditioned medium likely reflect a reduced rate in the secretion of functional fVIII molecules.

When fVIII production by $\mathrm{CHO}$ cells was evaluated in the presence of $\mathrm{vWf}$, the $\mathrm{vWf}$-dependent increase in accumulation of fVIII in the culture medium was reduced for the mutants compared with normal fVIII. These results support the concept that the reduced binding to $\mathrm{vWf}$ observed in plasma contributes to the low fVIII levels of the patients. Noteworthy, in this in vitro culture system, the addition of vWf resulted in only a 9-fold increase of the concentration of normal fVIII. This increase is in contrast to the situation of patients with severe vWD, in whom the fVIII levels are reduced more than 20 -fold. ${ }^{3}$ This reduction is likely due to the fact that clearance mechanisms or inactivation phenomena at play in vivo are absent or less effective in the in vitro cell culture system. Accordingly, it can be expected that the reduction of fVIII stabilization observed in vitro with Arg2150His and Ile2098Ser fVIII is even more significant in vivo.

These findings demonstrate that mutations in the fVIII C1 domain leading to a reduced fVIII binding to $\mathrm{vWf}$ without alteration of fVIII procoagulant activity are an important cause of mild/moderate hemophilia A.

\section{References}

1. Kemball-Cook G, Tuddenham EG. The factor VIII mutation database on the World Wide Web: the haemophilia A mutation, search, test and resource site. Hamsters update (version 3.0, http:// europium.csc.mrc.ac.uk). Nucleic Acids Res. 1997;25:128-132.

2. Vehar GA, Keyt B, Eaton D, et al. Structure of human factor VIII. Nature. 1984;312:337-342.

3. Weiss HJ, Sussman II, Hoyer LW. Stabilization of factor VIII in plasma by the von Willebrand factor: studies on posttransfusion and dissociated factor
VIII and in patients with von Willebrand's disease. J Clin Invest. 1997;60:390-404.

4. Jorieux S, Tuley EA, Gaucher C, et al. The mutation $\operatorname{Arg}(53) \rightarrow \operatorname{Trp}$ causes von Willebrand disease Normandy by abolishing binding to factor VIII: studies with recombinant von Willebrand factor. Blood. 1992;79:563-567.

5. Kaufman RJ, Wasley LC, Dorner AJ. Synthesis, processing, and secretion of recombinant human factor VIII expressed in mammalian cells. J Biol Chem. 1988;263:6352-6362.
6. Wise R, Dorner A, Krane M, et al. The role of von Willebrand factor multimers and propeptide cleavage in binding and stabilization of factor VIII. J Biol Chem. 1991;266:21948-21955.

7. Koedam J, Meijers J, Sixma J, et al. Inactivation of human factor VIII by activated protein C: cofactor activity of protein $\mathrm{S}$ and protective effect of von Willebrand factor. J Clin Invest. 1988;82: 1236-1243.

8. Koedam JA, Hamer RJ, Beeser-Visser NH, et al. The effect of von Willebrand factor on activation 
of factor VIII by factor Xa. Eur J Biochem. 1990; 189:229-234.

9. Andersson LO, Brown JE. Interaction of facto VIII-von Willebrand factor with phospholipid vesicles. Biochem J. 1981;200:161-167.

10. Nesheim M, Pittman DD, Giles AR, et al. The effect of plasma von Willebrand factor on the binding of the human factor VIII to thrombin-activated platelets. J Biol Chem. 1991;266:17815-17820.

11. Gilbert GE, Drinkwater D, Barter S, et al. Specificity of phosphatidylserine-containing membrane binding sites for factor VIII. J Biol Chem. 1992; 267:15861-15868.

12. Hamer RJ, Koedam JA, Beeser-Visser NH, et al. Factor VIII binds to von Willebrand factor via its Mr 80000 light chain. Eur J Biochem. 1987;166 37-43.

13. Lollar P, Hill-Eubanks DC, Parker GC. Association of the factor VIII light chain with von Willebrand factor. J Biol Chem. 1988:263:10451. 10455.

14. Leyte A, Verbeet MP, Brodniewicz-Proba T, et al. The interaction between human blood coagulation factor VIII and von Willebrand factor. Biochem J. 1989;257:679-683.

15. Shima M, Scandella D, Yoshioka A, et al. A Factor VIII neutralizing monoclonal antibody and a human inhibitor alloantibody recognizing epitopes in the $\mathrm{C} 2$ domain inhibit factor VIII binding to von Willebrand factor and to phosphatidylserine. Thromb Haemost. 1993;69:240-243.

16. Saenko EL, Shima M, Rajalakshmi KJ, et al. A role for the C2 domain of factor VIII in binding to von Willebrand factor. J Biol Chem. 1994;269: 11601-11605.

17. Saenko EL, Scandella D. A mechanism for inhibition of factor VIII binding to phospholipid by von Willebrand factor. J Biol Chem. 1995;270:1382613833.
18. Saenko EL, Scandella D. The acidic region of the factor VIII light chain and the C2 domain together form the high affinity binding site for von Willebrand factor. J Biol Chem. 1997:272:1800718014.

19. Peerlinck K, Jacquemin M, Arnout J, et al. Antifactor VIII antibody inhibiting allogeneic but not autologous factor VIII in patients with mild hemophilia A. Blood. 1999;93:2267-2273.

20. Gilles JG, Lavend'homme R, Peerlinck K, et al. Some factor VIII (fVIII) inhibitors recognise a fVIII epitope(s) that is present only on fVIII-vWf complexes. Thromb Haemost. 1999;82:40-45.

21. Jacquemin M, Benhida A, Peerlinck K, et al. A human antibody directed to the factor VIII C1 domain inhibits factor VIII cofactor activity and binding to von Willebrand factor. Blood. 2000;95:156163.

22. Peerlinck K, Eikenboom JC, Ploos, et al. A patient with von Willebrand's disease characterized by a compound heterozygosity for a substitution of Arg854 by Gln in the putative factor-VIII-binding domain of von Willebrand factor (vWf) on one allele and very low levels of mRNA from the second vWf allele. Br J Haematol. 1992;80:358-363.

23. Chuah M, Brems H, Vanslembrouck V, et al. Bone marrow stromal cells as targets for gene therapy of hemophilia A. Hum Gene Ther. 1998;9:353 365.

24. Horton RM, LR Pease. Recombination and mutagenesis of DNA sequences using PCR. In: McPherson MJ, ed. Directed Mutagenesis: A Practical Approach. Oxford: IRL Press; 1991:217-228.

25. Pittman DD, Kaufman RJ. Site-directed mutagenesis and expression of coagulation Factors VIII and $\mathrm{V}$ in mammalian cells. Methods Enzymol. 1993:222:236-260.

26. Jacquemin MG, Desqueper BG, Benhida A, et al. Mechanisms and kinetics of factor VIII inactiva- tion: study with an IgG4 monoclonal antibody derived from a hemophilia $A$ patient with inhibitor. Blood. 1998;92:496-506.

27. Vlot AJ, Koppelman SJ, Meijers JC, et al. Kinetics of factor VIII-von Willebrand factor association. Blood. 1996;87:1809-1816.

28. Voorberg J, de Laaf RTM, Koster PM, et al. Intracellular retention of a factor VIII protein with an Arg2307 $\rightarrow$ Gln mutation as a cause of haemophilia A. Biochem J. 1996:318:931-937.

29. Pipe SW, Kaufman RJ. Factor VIII C2 domain missense mutations exhibit defective trafficking of biological functional proteins. J Biol Chem. 1996; 271:25671-25676.

30. Gitschier J, Wood WI, Shuman MA, et al. Identification of a missense mutation in the factor VIII gene of a mild hemophiliac. Science. 1986;232: 1415-1416.

31. Leyte A, van Schijndel HB, Niehrs C, et al. Sulphation of Tyr1680 of human blood coagulation factor VIII is essential for the interaction of factor VIII with von Willebrand factor. J Biol Chem. 1991;266:740-746.

32. Pittman DD, Wang JH, Kaufman RJ. Identification and functional importance of tyrosine sulfate residues within recombinant factor VIII. Biochemistry. 1992;31:3315-3325

33. Gale AJ, Pellequer JL, Getzoff ED, et al. Structural basis for hemophilia A caused by mutations in the $\mathrm{C}$ domains of blood coagulation Factor VIII. Thromb Haemost. 2000;83:78-85.

34. Jacquemin MG, Pellequer JL, Gale AJ, et al. Molecular mechanism of mild/moderate hemophilia A: substitution lle2098Ser in the Factor(FVIII) C1 domain impairs FVIII binding to von Willebrand factor without altering FVIII production [abstract]. Blood. 1999;94:372a. 